\title{
DAVID HOMEOMORPHISMS VIA CARLESON BOXES
}

\author{
Edson de Faria \\ University of São Paulo, Department of Mathematics \\ Rua do Matão 1010, CEP 05508-090, São Paulo, SP, Brazil; edson@ime.usp.br
}

\begin{abstract}
We construct a family of examples of increasing homeomorphisms of the real line whose local quasi-symmetric distortion blows up almost everywhere, which nevertheless can be realized as the boundary values of David homeomorphisms of the upper half-plane. The construction of such David extensions uses Carleson boxes.
\end{abstract}

\section{Introduction}

This paper answers a question that was posed by Zakeri, in conversation with the author, some time ago. The question concerns David homeomorphisms, which are very useful generalizations of quasi-conformal mappings introduced by David in [D] (see below for the definition). Such homeomorphisms were used for the first time in complex dynamics by Haïssinsky $[\mathrm{H}]$ to perform parabolic surgery on rational maps, and were later used by Petersen and Zakeri [PZ] in their study of quadratic polynomials with Siegel disks.

The question asked by Zakeri can be roughly stated as follows. Suppose a given homeomorphism of the real line is the boundary map of a David homeomorphism of the upper half-plane. How bad can its pointwise distortion be? Before we can explain the meaning and give the precise statement of Zakeri's question, we need to recall some standard notions.

1.1. Quasi-conformality. Let $f: U \rightarrow V$ be an orientation-preserving homeomorphism between open subsets of the plane. If $f$ is differentiable at $z \in U$ with $\partial f(z) \neq 0$, its Beltrami coefficient at $z$ is $\mu_{f}(z)=\bar{\partial} f(z) / \partial f(z)$. The homeomorphism $f=u+i v$ is said to be absolutely continuous on lines (ACL) if the restrictions of $u$ and $v$ (the real and imaginary parts of $f$, respectively) to Lebesgue almost every horizontal and Lebesgue almost every vertical lines are absolutely continuous functions. The ACL condition implies differentiability almost everywhere. We say that $f$ is quasi-conformal if $f$ is ACL and its Beltrami coefficient $\mu_{f}(z)$ (defined almost everywhere) satisfies $\left|\mu_{f}(z)\right| \leq k<1$ a.e., for some constant $0 \leq k<1$. The dilatation or conformal distortion of $f$ at $z$ is

$$
K_{f}(z)=\frac{1+\left|\mu_{f}(z)\right|}{1-\left|\mu_{f}(z)\right|}
$$

doi:10.5186/aasfm.2011.3613

2010 Mathematics Subject Classification: Primary 30C62; Secondary 37F30, 26A30.

Key words: David homeomorphism, Carleson boxes, quasi-symmetric distortion, Borel-Cantelli argument.

This work received the financial support of FAPESP through Projeto Temático Dinâmica em Baixas Dimensões. 
The supremum of $K_{f}(z)$ over all $z$ is called the maximal dilatation, denoted $K_{f}$. Note that $K_{f}<\infty$ whenever $f$ is quasi-conformal. The composition $f \circ g$ of quasiconformal homeomorphisms $f, g$ is quasi-conformal, and $K_{f \circ g} \leq K_{f} K_{g}$. See [A] or $[\mathrm{G}]$ for these facts on qc-mappings, and much more.

1.2. David homeomorphisms. The concept of David homeomorphism, introduced by David in [D], is a generalization of quasi-conformality. One allows the dilatation of $f$ to degenerate - in other words, one allows $K_{f}(z) \rightarrow \infty$ at certain places - but in a controlled fashion. More precisely, a homeomorphism $f: U \rightarrow V$ between open sets of the plane is David if $f$ is orientation-preserving, ACL, and there exist constants $C>0$ and $\alpha>0$ such that, for each $\lambda>0$ we have

$$
\text { Area }_{s}\left\{z \in U: K_{f}(z)>\lambda\right\}<C e^{-\alpha \lambda} \text {. }
$$

Here Area $_{s}$ denotes the spherical area, namely

$$
\operatorname{Area}_{s}(E)=\iint_{E} \frac{d x d y}{\left(1+|z|^{2}\right)^{2}},
$$

for every Borel measurable set $E \subseteq \mathbf{C}$. When $U$ is bounded, we may safely replace the spherical area by the usual Euclidean area (two-dimensional Lebesgue measure) in the definition.

1.3. Quasi-symmetric distortion. Given an increasing homeomorphism of the real line $\phi: \mathbf{R} \rightarrow \mathbf{R}$ we define its quasi-symmetric local distortion function to be

$$
\sigma_{\phi}(x)=\lim \sup _{t \downarrow 0} \max \left\{\frac{\phi(x+t)-\phi(x)}{\phi(x)-\phi(x-t)}, \frac{\phi(x)-\phi(x-t)}{\phi(x+t)-\phi(x)}\right\} .
$$

We say that $\phi$ is quasi-symmetric if there exists $M \geq 1$ such that

$$
\frac{1}{M} \leq \frac{\phi(x+t)-\phi(x)}{\phi(x)-\phi(x-t)} \leq M
$$

for all $x \in \mathbf{R}$ and all $t>0$. In particular, if $\phi$ is quasi-symmetric with quasisymmetry constant $M$, then $\sigma_{\phi}(x) \leq M$ for all $x$. If $\phi$ is quasi-symmetric, then it can be extended to a quasi-conformal homeomorphism of the upper half-plane (and the dilatation of the extension can be bounded in terms of the quasi-symmetry constant of $\phi$ only). Conversely, the boundary values of every quasi-conformal homeomorphism of the upper half-plane are given by a quasi-symmetric homeomorphism of the real line. These facts are well-known, and their proofs can be found in [A].

Following [Z], we define the pointwise distortion of $\phi$ at $x \in \mathbf{R}$, denoted by $\lambda_{\phi}(x)$, to be the analogue of $(1)$, with ' $\sup _{t>0}$ ' replacing 'lim $\sup _{t \rightarrow 0}$ '. Thus, $\sigma_{\phi}(x) \leq$ $\lambda_{\phi}(x)$ for all $x$. It is proved in [Z, Thm. A] that, if $\phi: \mathbf{R} \rightarrow \mathbf{R}$ is an increasing homeomorphism with $\phi(x+1)=\phi(x)$ for all $x$ and $\lambda_{\phi} \in L^{p}[0,1]$ for some $p>0$, then $\phi$ extends to a David homeomorphism $f$ of the upper half-plane (still satisfying $f(z+1)=f(z)$ for all $z)$. This integrability condition is sufficient, but not necessary, for David extendibility. It implies, of course, that $\lambda_{\phi}$ is almost everywhere finite. The question arises: Is it possible for the pointwise distortion $\lambda_{\phi}$ to blow up in a large subset of the real line and yet $\phi$ still be extendible to a David homeomorphism? This is the question asked by Zakeri, and our answer is given by the following result.

Theorem 1. There exists a family of increasing homeomorphisms $\phi: \mathbf{R} \rightarrow \mathbf{R}$ having the following properties. 
(a) $\sigma_{\phi}(x)=\infty$ for every $x$ in a full-measure subset of $\mathbf{R}$;

(b) $\phi$ has modulus of continuity $\rho(t)=t \log (1 / t)$;

(c) $\phi=\left.f\right|_{\mathbf{R}}$, where $f$ is a David homeomorphism of the (closed) upper half-plane.

Note that, although the pointwise distortions of the examples produced by this theorem blow up almost everywhere, these homeomorphisms still exhibit some regularity, in the sense that they are $\alpha$-Hölder continuous for all $0<\alpha<1$ (as implied by (b)). In order to prove this theorem, we will first show that, given an increasing homeomorphism $\phi$ of the real line satisfying some suitable conditions, a David extension of $\phi$ to the upper half-plane can be constructed using a system of Carleson boxes. We will then verify that these conditions are sufficiently mild to allow for a $\phi$ with properties (a) and (b) above. Apart from an unavoidable limit, the construction of such $\phi$ 's follows a rather explicit discrete, piecewise-linear scheme. The proof that (a) holds in our examples will involve a simple Borel-Cantelli argument.

Remark 1. Each example $\phi$ to be constructed in the proof of theorem 1 will be the lift of a circle homeomorphism (i.e., it will satisfy $\phi(x+1)=\phi(x)+1$ for all $x$ ), and its David extension $f$ will satisfy $f(z+1)=f(z)+1$ for all $z$ in the upper half-plane. Therefore everything can be quotiented down to the unit disk to produce examples of David homeomorphisms of $\mathbf{D}$ with pointwise distortion blowing up almost everywhere on $\mathbf{S}^{1}=\partial \mathbf{D}$.

\section{Dyadic approximations}

Let $\phi:[0,1] \rightarrow[0,1]$ be an increasing homeomorphism. We associate to $\phi$ a sequence $\phi_{n}:[0,1] \rightarrow[0,1]$ of dyadic approximations defined as follows. Denote by $\mathscr{P}_{n}$ the $n$-th dyadic partition of $[0,1]$ (modulo endpoints), namely

$$
\mathscr{P}_{n}=\left\{\left[\frac{j}{2^{n}}, \frac{j+1}{2^{n}}\right]: 0 \leq j \leq 2^{n}-1\right\} .
$$

Its elements are called atoms, and the endpoints of atoms are called vertices. Each atom of $\mathscr{P}_{n}$ is the union of exactly two atoms of $\mathscr{P}_{n+1}$, for all $n \geq 0$. We define $\phi_{n}:[0,1] \rightarrow[0,1]$ so that $\phi_{n}$ is affine on each atom of $\mathscr{P}_{n}$ and so that it agrees with $\phi$ in the vertices of $\mathscr{P}_{n}$ (i.e., the points $j 2^{-n}, 0 \leq j \leq 2^{n}-1$ ). Note in particular that $\phi_{0}=\mathrm{id}$ and that each $\phi_{n}$ is quasi-symmetric. Moreover, $\phi_{n}(\Delta)=\phi(\Delta)$ for each $\Delta \in \mathscr{P}_{n}$. More importantly, the sequence of piecewise linear homeomorphisms $\phi_{n}$ converges uniformly to $\phi$. For later use, let us define a sequence of positive numbers $M_{1}, M_{2}, \ldots$ as follows:

$$
M_{n}=\sup \frac{\left|\phi_{n}(\Delta)\right|}{\left|\phi_{n}\left(\Delta^{*}\right)\right|},
$$

where the supremum is taken over all pairs of atoms $\Delta, \Delta^{*} \in \mathscr{P}_{n}$ lying in a single atom of $\mathscr{P}_{n-1}$ (i.e., such that $\Delta \cup \Delta^{*} \in \mathscr{P}_{n-1}$ ). Note that $M_{n} \geq 1$; in fact $M_{n}>1$ unless $\phi_{n}=\phi_{n-1}$. We define the cumulative distortion function of $\phi$ to be the function $\kappa_{\phi}: \mathbf{N} \rightarrow \mathbf{R}^{+}$given by

$$
\kappa_{\phi}(n)=\prod_{j=1}^{n} \frac{1+M_{j}}{2} .
$$


Let us also remark that the slope $s_{\Delta}$ of $\phi_{n}$ on each atom $\Delta \in \mathscr{P}_{n}$ satisfies

$$
\prod_{j=1}^{n} \frac{2}{1+M_{j}} \leq s_{\Delta} \leq \prod_{j=1}^{n} \frac{2 M_{j}}{1+M_{j}} .
$$

The following result is an easy consequence of the definitions given so far, and will be used in $\S 3$.

Lemma 1. For each $n \geq 1$, the piecewise affine homeomorphism $\phi_{n} \times \mathrm{id}:[0,1] \times$ $\mathbf{R} \rightarrow[0,1] \times \mathbf{R}$ has quasi-conformal distortion bounded by $\kappa_{\phi}(n)$.

Proof. If $h(x)=a x+b$ is an affine homeomorphism of the line with $a>0$, then $H=h \times$ id is quasi-conformal and $K_{H} \leq \max \left\{a, a^{-1}\right\}$, as the reader can easily check. The lemma follows from this fact and the estimate (3), taking into account that

$$
\max \left\{s_{\Delta}, s_{\Delta}^{-1}\right\} \leq \prod_{j=1}^{n} \frac{1+M_{j}}{2}=\kappa_{\phi}(n) .
$$

\section{Carleson boxes extensions}

Assume we are given $\phi$ as in the previous section, and its associated sequence $\left(\phi_{n}\right)$ of dyadic approximations. Let us define an extension of $\phi$ to a homeomorphism $f:[0,1]^{2} \rightarrow[0,1]^{2}$, using a Carleson boxes construction in order to bound its local quasi-conformal distortion. We will define $f$ as a limit

$$
f=\lim _{n \rightarrow \infty} f_{n} \circ f_{n-1} \circ \cdots \circ f_{1}
$$

in the $C^{0}$-topology, where each $f_{n}$ is a piecewise affine homeomorphism of the unit square built from $\phi_{n}$.

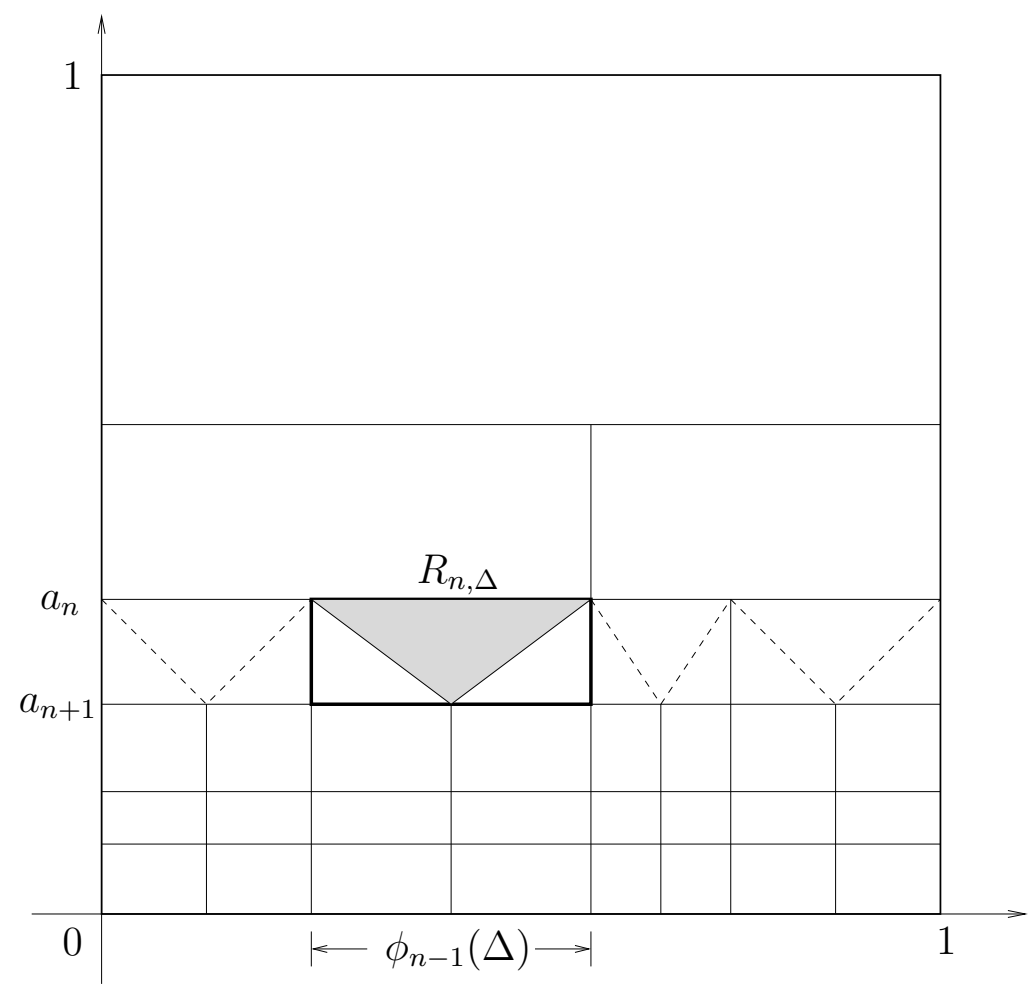

Figure 1. Images of Carleson boxes under $f^{(n-1)}$. 
Here is the detailed description. Let us fix a sequence $1=a_{0}>a_{1}>a_{2}>$ $\cdots>a_{n}>\cdots$ of positive numbers converging to zero. We define $f_{n}:[0,1]^{2} \rightarrow[0,1]^{2}$ to be equal to the identity map on the set $\left\{(x, y) \in[0,1]^{2}: y \geq a_{n}\right\}$, to be equal to $\left(\phi_{n} \circ \phi_{n-1}^{-1}\right) \times$ id on the set $\left\{(x, y) \in[0,1]^{2}: 0 \leq y<a_{n+1}\right\}$, and interpolating in a piecewise linear fashion between these two on the strip $[0,1] \times\left[a_{n+1}, a_{n}\right]$. This interpolating map $g_{n}:[0,1] \times\left[a_{n+1}, a_{n}\right] \hookleftarrow$ in turn is defined as follows. Divide each rectangle of the form $\phi_{n-1}(\Delta) \times\left[a_{n+1}, a_{n}\right]$, with $\Delta \in \mathscr{P}_{n-1}$, into 3 triangles, by joining the two upper-most vertices of the rectangle to the midpoint of its bottom side, in the domain of $g_{n}$ (see Figure 1). Similarly, in the target, divide each rectangle $\phi_{n-1}(\Delta) \times\left[a_{n+1}, a_{n}\right]$ into 3 triangles by joining the upper-most vertices to the point on the bottom side that lies vertically above the image of the midpoint of $\phi_{n-1}(\Delta)$ under $\phi_{n} \circ \phi_{n-1}^{-1}$. Let $g_{n}$ map each triangle in the domain affinely onto the corresponding triangle in the target, in the obvious way (see Figure 1). Summarizing, the piecewise affine homeomorphism $f_{n}$ has been defined so that

$$
f_{n}(x, y)= \begin{cases}(x, y) & \text { if } y \geq a_{n} \\ g_{n}(x, y) & \text { if } a_{n+1}<y<a_{n} \\ \left(\phi_{n} \circ \phi_{n-1}^{-1}(x), y\right) & \text { if } 0 \leq y \leq a_{n+1}\end{cases}
$$

Note that the composition $f^{(n)}=f_{n} \circ f_{n-1} \circ \cdots \circ f_{1}$ agrees with $\phi_{n} \times$ id on the strip $[0,1] \times\left[0, a_{n+1}\right]$. Moreover, for each $(x, y) \in[0,1]^{2}$ with $y>0$, the sequence $f^{(n)}(x, y)$ is eventually constant. The limiting homeomorphism $f=\lim f^{(n)}$ agrees with $\phi=\lim \phi_{n}$ on $[0,1] \times\{0\}$. We have also $f(x, y)=f^{(n)}(x, y)$ for all $(x, y) \in$ $[0,1] \times\left[a_{n+1}, a_{n}\right]$.

Definition 1. The homeomorphism $f$ constructed above is called a Carleson boxes extension of $\phi$.

Note that the restriction of $f$ to each strip of the form $[0,1] \times\left[a_{n+1}, a_{n}\right]$ is piecewise affine, hence quasi-conformal. We wish to bound the local dilatation of $f$ in each strip. For this purpose, we decompose the unit square (in the domain) into Carleson boxes

$$
B_{n, j}=\left[\frac{j}{2^{n}}, \frac{j+1}{2^{n}}\right] \times\left[a_{n+1}, a_{n}\right], \quad n \geq 0,0 \leq j \leq 2^{n}-1 .
$$

The first thing to notice is that the qc-distortion of $f^{(n)}$ on each box $B_{m, j}$ with $m>n$ is bounded by $\kappa_{\phi}(n)$, the cumulative distortion function introduced in (2). This is immediate from Lemma 1. In order to bound the qc-distortion of $f$, we therefore still need an estimate on $K_{f^{(n)}}(z)$ for all $z \in B_{j, n}$. Here we have to remember that $f^{(n)}=f_{n} \circ f^{(n-1)}$, and that $f_{n}$ agrees with $g_{n}$ on the strip $[0,1] \times\left[a_{n+1}, a_{n}\right]$. This strip has been decomposed into triangles of two types: upside-down triangles, such as the one shaded in Figure 1, and those with one side supported in the bottom edge of the strip. In the latter triangles, $g_{n}$ agrees with $\left(\phi_{n} \circ \phi_{n-1}^{-1}\right) \times$ id. Hence in those triangles $f^{(n)}$ agrees with $g_{n} \circ f^{(n-1)}=\phi_{n} \times \mathrm{id}$, and therefore (again by Lemma 1 ) its qc-distortion there is bounded by $\kappa_{\phi}(n)$. In the upside-down triangles, $g_{n}$ is a linear shearing map. Hence we need the following lemma. 
Lemma 2. Let $T: \mathbf{R}^{2} \rightarrow \mathbf{R}^{2}$ be the linear shearing map given by $T(x, y)=$ $(x+b y, y)$, where $b>0$. Then its qc-dilatation is everywhere constant, equal to

$$
K_{T}=\left(\frac{b}{2}+\sqrt{1+\frac{b^{2}}{4}}\right)^{2} .
$$

Proof. Writing $T$ in complex coordinates, we get

$$
T(z, \bar{z})=\left(1+\frac{b}{2 i}\right) z-\frac{b}{2 i} \bar{z}
$$

Hence

and therefore

$$
\partial T=1+\frac{b}{2 i}, \quad \bar{\partial} T=-\frac{b}{2 i},
$$

This gives us

$$
k=\left|\frac{\bar{\partial} T}{\partial T}\right|=\frac{b / 2}{\sqrt{1+b^{2} / 4}} .
$$

from which (5) follows.

$$
K_{T}=\frac{1+k}{1-k}=\frac{b / 2+\sqrt{1+b^{2} / 4}}{\sqrt{1+b^{2} / 4}-b / 2}
$$

Remark 2. Note that the same result holds true if $T$ is post-composed with a translation. Note also that for $b$ large $(b>1)$ the right-hand side of $(5)$ is $O\left(b^{2}\right)$, whereas for $b$ small $(b<1)$, it is simply $O(1)$.

To apply lemma 2 in our situation, each rectangle

$$
R_{n, \Delta}=\phi_{n-1}(\Delta) \times\left[a_{n+1}, a_{n}\right]
$$

should be normalized to unit size by a suitable affine map. We note en passant that $R_{n, \Delta}=f^{(n-1)}\left(B_{n-1, j}\right)$ for some $j$. Let us denote by $U_{n, \Delta} \subset R_{n, \Delta}$ the upside-down triangle determined by the top side of $R_{n, \Delta}$ and the midpoint of its bottom side (see Figure 2). We want to bound the qc-distortion of $g_{n}$ on $U_{n, \Delta}$, and the result we need is lemma 3 below. The rough idea is that such distortion will be bounded provided the rectangles $R_{n, \Delta}$ don't become too skinny. As we shall see, if we choose the sequence $a_{n}$ so that $\delta_{n}=a_{n}-a_{n+1}$ decreases to zero at a slower rate than $2^{-n}$, then the map $g_{n}$ restricted to the upside-down triangle $U_{n, \Delta}$ will be a linear shearing with small $b$, whose qc-distortion is $O(1)$ by lemma 2 and the above remark.

Let us agree to say that a non-decreasing sequence $\tau: \mathbf{N} \rightarrow \mathbf{R}^{+}$has sub-exponential growth if

$$
\lim _{n \rightarrow \infty} \frac{\log \tau(n)}{n}=0
$$

For instance, if $\tau(n)$ grows at most polynomially with $n$, then $\tau$ has sub-exponential growth. This is tantamount to saying that $\tau(n) \rho^{-n} \rightarrow 0$ for all $\rho>1$. We have the following lemma.

Lemma 3. Suppose $\rho=\liminf \left(2^{n} \delta_{n}\right)>1$, and assume also that $\kappa_{\phi}$ has subexponential growth. Then $K_{\left.g_{n}\right|_{U_{n}}}=O(1)$, i.e., the qc-distortion of $g_{n}$ in each upside-down triangle $U_{n, \Delta}$ is bounded, and uniformly so in $n$. 
$Q$

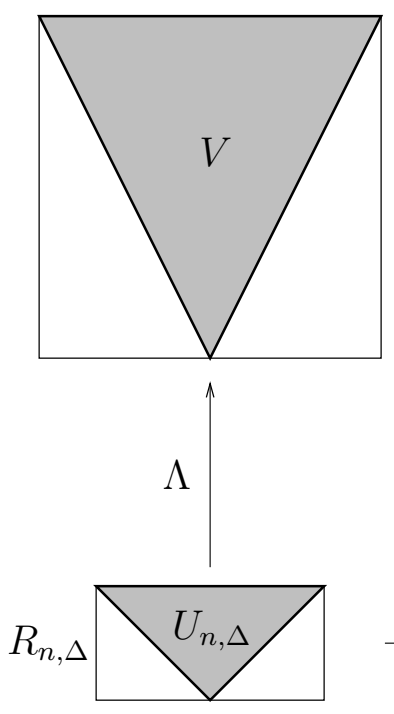

$Q$

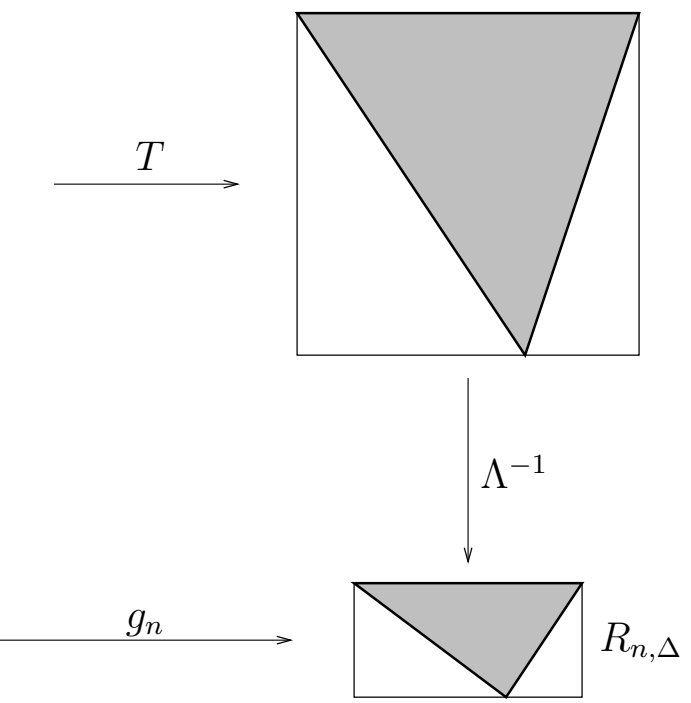

Figure 2. Normalizing the shearing map $g_{n}$.

Proof. We denote by $Q$ the unit square $[0,1]^{2}$. Let $\Lambda: R_{n, \Delta} \rightarrow Q$ be the affine orientation-preserving map sending vertices to vertices (and preserving horizontals). Since the horizontal sides of $R_{n, \Delta}$ have length $\ell_{n}=\left|\phi_{n-1}(\Delta)\right|$ and its vertical sides have length $\delta_{n}$, the map $\Lambda$ map has the form

$$
\Lambda(x, y)=\left(\ell_{n}^{-1} x+\alpha, \delta_{n}^{-1} y+\beta\right) .
$$

Here the constants $\alpha, \beta$ depend on $n, \Delta$, of course, but their actual values are irrelevant to us. Since $g_{n}\left(R_{n, \Delta}\right)=R_{n, \Delta}$, we can write $\left.g_{n}\right|_{U_{n, \Delta}}=\Lambda^{-1} \circ T \circ \Lambda$, where $T$ is a linear shearing map of the form $T(x, y)=(x+b y+c, y)$, where $b=O(1)$ (in fact, one can easily check that $b \leq 1 / 2$; see Figure 2$)$. A simple calculation yields, for each $(x, y) \in U_{n, \Delta}$,

$$
g_{n}(x, y)=\left(x+b \ell_{n} \delta_{n}^{-1} y+A, y+B\right),
$$

where $A, B$ are constants. Now, taking into account that $|\Delta|=2^{-n+1}$, and using (3) and (4) of lemma 1, we see that

$$
\ell_{n}=\left|\phi_{n-1}(\Delta)\right|=s_{\Delta}|\Delta| \leq \kappa_{\phi}(n) 2^{-n+1} .
$$

This shows that

$$
b \ell_{n} \delta_{n}^{-1} \leq 2 b \kappa_{\phi}(n)\left(2^{n} \delta_{n}\right)^{-1} \leq 2 b \kappa_{\phi}(n) \rho^{-n} \rightarrow 0
$$

as $n \rightarrow \infty$, because $\kappa_{\phi}$ has sub-exponential growth. Taking this information back to (6) and applying lemma 2, we get the desired result.

Combining all of the above facts, we arrive at the following lemma.

Lemma 4. Suppose $\lim \inf \left(2^{n} \delta_{n}\right)>1$, and assume also that $\kappa_{\phi}(n)$ has subexponential growth. Then the maximal dilatation $K_{n, j}$ of $f$ in each Carleson box $B_{n, j}$ satisfies the inequality $K_{n, j} \leq c \kappa_{\phi}(n)$, for some uniform constant $c>0$.

Now we have the following theorem, which is the main result of this section.

Theorem 2. Let $\phi:[0,1] \rightarrow[0,1]$ be an increasing homeomorphism whose cumulative distortion function $\kappa_{\phi}(n)$ satisfies $\kappa_{\phi}(n) \leq C n$ for some $C>0$. Then $\phi$ extends to a David homeomorphism of the unit square. 
Proof. Consider the Carleson boxes extension $f$ constructed above. We assume that the sequence $\delta_{n}=a_{n}-a_{n+1}$ is chosen to be of the form $\delta_{n}=c_{0} \delta^{n}$ for some $\frac{1}{2}<\delta<1$, so that the hypothesis of lemma 4 is satisfied. Let us estimate the area of the set of points where $K_{f}$ is greater than $\lambda$ for any given $\lambda>0$. Using lemma 4 , we have

$$
\begin{aligned}
\text { Area }\left\{z: K_{f}(z)>\lambda\right\} & \leq \operatorname{Area}\left\{\bigcup_{K_{n, j}>\lambda} B_{n, j}\right\} \leq \operatorname{Area}\left\{\bigcup_{\kappa_{\phi}(n)>c^{-1} \lambda} \bigcup_{j=0}^{2^{n}-1} B_{n, j}\right\} \\
& =\sum_{\kappa_{\phi}(n)>c^{-1} \lambda}\left(\sum_{j=0}^{2^{n}-1} \operatorname{Area}\left(B_{n, j}\right)\right)=\sum_{\kappa_{\phi}(n)>c^{-1} \lambda} \delta_{n} .
\end{aligned}
$$

Now, suppose we know that, for all $m \geq 1$ and constants $C_{1}, \alpha>0$,

$$
\sum_{n \geq m} \delta_{n} \leq C_{1} e^{-\alpha \kappa_{\phi}(m)} .
$$

Then, letting $m=\inf \left\{n: \kappa_{\phi}(n)>c^{-1} \lambda\right\}$, we see from (7) that

$$
\text { Area }\left\{z: K_{f}(z)>\lambda\right\} \leq C_{1} e^{-\alpha \kappa_{\phi}(m)}<C_{1} e^{-\alpha \lambda / c},
$$

and therefore $f$ is indeed a David homeomorphism.

Hence, all we need is that (8) be true. But on the one hand, since $\delta_{n}=c_{0} \delta^{n}$, the left-hand side of (8) is

$$
\sum_{n \geq m} \delta_{n}=\frac{c_{0} \delta^{m}}{1-\delta}=\frac{c_{0}}{1-\delta} e^{-m \log \frac{1}{\delta}} .
$$

On the other hand, since $\kappa_{\phi}(n)<C n$, the right-hand side of (8) is bounded from below by $e^{-\alpha C m}$. Therefore (8) will be true provided we take $\alpha=C^{-1} \log \frac{1}{\delta}$ and $C_{1}=(1-\delta) / c_{0}$.

\section{Divergent quasi-symmetric distortion}

Let us now define a special family of homeomorphisms of the unit interval, whose members have divergent local quasi-symmetric distortion almost everywhere. Each member of the family will be determined by a given sequence of quasi-symmetric weights $\mathscr{M}=\left(M_{1}, M_{2}, \ldots, M_{n}, \ldots\right)$, where $M_{n}>1$ for each $n \geq 1$, and will accordingly be denoted by $\phi_{\mathscr{M}}$. We define $\phi_{\mathscr{M}}$ as the limit of a sequence of dyadic piecewise linear homeomorphisms $\phi_{n}$, inductively constructed as follows. We take $\phi_{0}=$ id. Suppose $\phi_{n}$ has been defined so that it is linear on each atom of $\mathscr{P}_{n}$. Given an atom $\Delta \in \mathscr{P}_{n}$, write $\Delta=\Delta^{-} \cup \Delta^{+}$where $\Delta^{-}, \Delta^{+} \in \mathscr{P}_{n+1}$ and $\Delta^{-}$lies to the left of $\Delta^{+}$. Define $\phi_{n+1}$ on $\Delta$ so that it agrees with $\phi_{n}$ at the endpoints of $\Delta$, is linear with slope $s^{-}$on $\Delta^{-}$, linear with slope $s^{+}$on $\Delta^{+}$, and $s^{-}=M_{n+1} s^{+}$(see Figure 3). Note that the arithmetic mean of the slopes $s^{-}, s^{+}$is equal to the slope $s_{\Delta}$ of $\phi_{n}$ on $\Delta$, so that

$$
s^{-}=\frac{2 M_{n+1}}{1+M_{n+1}} s_{\Delta} \quad \text { and } \quad s^{+}=\frac{2}{1+M_{n+1}} s_{\Delta} .
$$

Note also that, inductively defined in this way, the sequence $\left(\phi_{n}\right)$ satisfies $\phi_{n} \leq \phi_{n+1}$ for all $n$. We let $\phi_{\mathscr{M}}=\lim \phi_{n}$. This limit is uniform, i.e., it holds in the $C^{0}$ topology. Under suitable conditions on $\mathscr{M}$, the limiting map is better than continuous, as the following result shows. 


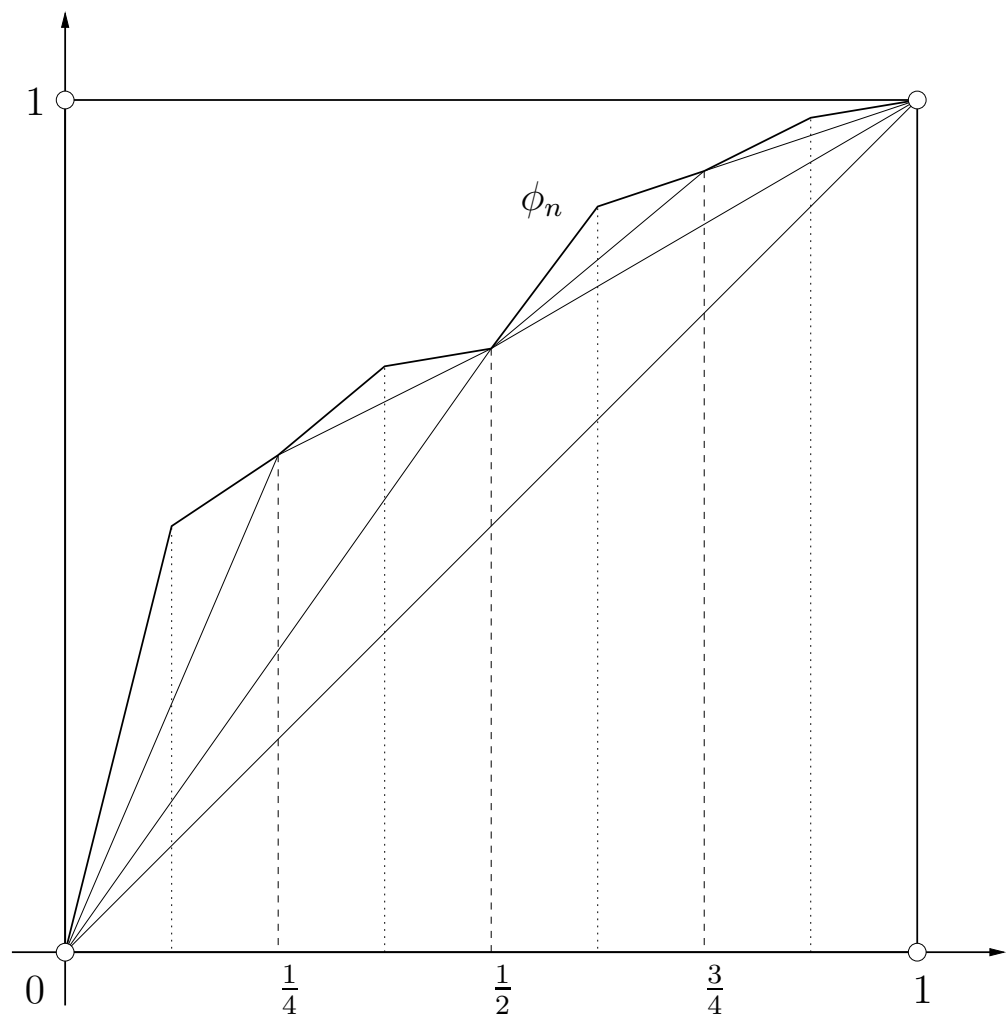

Figure 3. Dyadic approximations $\phi_{n}$ to $\phi$.

Lemma 5. Let $\mathscr{M}=\left(M_{n}\right)_{n \geq 1}$ be a sequence of quasi-symmetric weights such that $\prod_{j=1}^{n} M_{j} \leq \mathrm{cn}$ for all $n$ (and some constant $c>0$ ). Then $\phi_{\mathscr{M}}$ has modulus of continuity $\rho(t)=t \log (1 / t)$. In particular, it is $\alpha$-Hölder for every $0<\alpha<1$.

Proof. Let us write $\phi=\phi_{\mathscr{M}}$ in this proof. Given $x, y \in[0,1]$, say with $x<y$, let $n \geq 0$ be the smallest integer such that $[x, y] \supseteq \Delta_{x, y}$ for some $\Delta_{x, y} \in \mathscr{P}_{n}$. Then $[x, y]$ is contained in the union of at most three atoms of $\mathscr{P}_{n}$, and so $\left|\Delta_{x, y}\right| \leq|x-y| \leq$ $3\left|\Delta_{x, y}\right|$. Since $\left|\Delta_{x, y}\right|=2^{-n}$, this can be rewritten as

$$
c_{1}^{-1} n \leq \log \frac{1}{|x-y|} \leq c_{1} n
$$

for some $c_{1}>1$. Note that for each atom $\Delta \in \mathscr{P}_{n}$ we have $\phi(\Delta)=\phi_{n}(\Delta)$, and $\left|\phi_{n}(\Delta)\right|=s_{\Delta}|\Delta|$, where $s_{\Delta}$ is the slope of $\left.\phi_{n}\right|_{\Delta}$, But clearly (see (9))

$$
s_{\Delta} \leq \prod_{j=1}^{n} \frac{2 M_{j}}{1+M_{j}} .
$$

Combining these facts, we see that

$$
\begin{aligned}
|\phi(x)-\phi(y)| & \leq 3 \prod_{j=1}^{n} \frac{2 M_{j}}{1+M_{j}}\left|\Delta_{x, y}\right|=3 \prod_{j=1}^{n} \frac{M_{j}}{1+M_{j}} \\
& =3\left(\prod_{j=1}^{n} \frac{1}{1+M_{j}}\right)\left(\prod_{j=1}^{n} M_{j}\right) \leq 3 c \frac{n}{2^{n}} .
\end{aligned}
$$


But then, using (10), we deduce that

$$
|\phi(x)-\phi(y)| \leq C|x-y| \log \frac{1}{|x-y|},
$$

for some $C>0$, and so the lemma is proved.

Let us now convince ourselves that, under a simple condition on $\mathscr{M}$, the local quasi-symmetric distortion of $\phi_{\mathscr{M}}$ blows up at every dyadic rational. In what follows, we denote by $D^{+} h(x)$ and $D^{-} h(x)$ the right and left derivatives of $h$ at $x$ respectively. These Dini derivatives certainly exist at all points when $h$ is piecewise linear.

Lemma 6. Let $\left(\phi_{n}\right)$ be the defining sequence of dyadic approximations of $\phi=$ $\phi_{\mathscr{M}}$. If $\xi \in[0,1]$ has the form $\xi=p 2^{-n}$ with $p$ odd, then for all $k \geq 1$ we have

$$
\frac{D^{+} \phi_{n+k}(\xi)}{D^{-} \phi_{n+k}(\xi)}=\frac{1}{M_{n}} \prod_{j=1}^{k} M_{n+j} .
$$

In particular, if $\prod_{j=1}^{\infty} M_{j}=\infty$, then $\sigma_{\phi}(\xi)=\infty$.

Proof. This follows at once from the following facts which are immediate from the construction of $\phi$. First, we have on the one hand

$$
\frac{D^{+} \phi_{n}(\xi)}{D^{-} \phi_{n}(\xi)}=\frac{1}{M_{n}}
$$

On the other hand, when we construct $\phi_{j+1}$ from $\phi_{j}$, we replace each linear piece by two new ones; the slope of the new one on the left is multiplied by $2 M_{j} /\left(1+M_{j}\right)$, while the slope of the new piece on the right is multiplied by $2 /\left(1+M_{j}\right)$. Thus, working inductively we see that for each $k \geq 1$

$$
D^{+} \phi_{n+k}(\xi)=\prod_{j=1}^{k} \frac{2 M_{n+j}}{1+M_{n+j}} D^{+} \phi_{n}(\xi) .
$$

Likewise, we have

$$
D^{-} \phi_{n+k}(\xi)=\prod_{j=1}^{k} \frac{2}{1+M_{n+j}} D^{+} \phi_{n}(\xi) .
$$

Dividing (12) by (13) we get (11) as desired. Finally, for each $k \geq 1$ let $\Delta_{k}$ and $\Delta_{k}^{*}$ be the two atoms of $\mathscr{P}_{n+k}$ that have $\xi$ as their common vertex, with $\Delta_{k}^{*}$ on the left of $\Delta_{k}$. Then

$$
\left|\phi\left(\Delta_{k}\right)\right|=\left|\phi_{n+k}\left(\Delta_{k}\right)\right|=D^{+} \phi_{n+k}(\xi)\left|\Delta_{k}\right|
$$

and similarly

$$
\left|\phi\left(\Delta_{k}^{*}\right)\right|=\left|\phi_{n+k}\left(\Delta_{k}^{*}\right)\right|=D^{-} \phi_{n+k}(\xi)\left|\Delta_{k}\right|,
$$

where we have used that $\left|\Delta_{k}^{*}\right|=\left|\Delta_{k}\right|$. Therefore, by (11),

$$
\frac{\left|\phi\left(\Delta_{k}\right)\right|}{\left|\phi\left(\Delta_{k}^{*}\right)\right|}=\frac{1}{M_{n}} \prod_{j=1}^{k} M_{n+j} .
$$

Letting $k \rightarrow \infty$, we deduce that $\sigma_{\phi}(\xi)=\infty$. 
Before we proceed, we need to introduce a special class of sequences of quasisymmetric weights which, for lack of a better name, we call sparsely-jumping sequences. The ingredients in the construction of such a sequence are two other auxiliary sequences of positive numbers, $\left(u_{n}\right)$ and $\left(\theta_{n}\right)$. They must satisfy the following conditions.

(i) We have $1<u_{n} \leq 2$ for all $n$ and $C_{0}=\prod_{n=1}^{\infty} u_{n}<\infty$ (for example, we can take $\left.u_{n}=1+n^{-2}\right)$.

(ii) There exists a constant $C_{1}>1$ such that $C_{1}^{-1} \log n \leq \theta_{n} \leq C_{1} \log n$ for all $n$; in particular $\prod_{n=1}^{\infty} \theta_{n}=\infty$.

Given the above ingredients satisfying these two conditions, let us write $r_{n}=\theta_{1} \theta_{2} \cdots \theta_{n}$ and $k_{n}=\left\lceil r_{n}\right\rceil$. By discarding the first few terms if necessary, we may assume that $k_{1}<k_{2}<\ldots$. Note that $k_{n+1} / k_{n} \rightarrow \infty$ as $n \rightarrow \infty$. Now define $M_{n}$ as follows

$$
M_{n}= \begin{cases}u_{n} & \text { if } n \notin\left\{k_{1}, k_{2}, \ldots\right\} \\ \theta_{m} u_{k_{m}} & \text { if } n=k_{m}\end{cases}
$$

Definition 2. A sequence $\mathscr{M}=\left(M_{n}\right)$ constructed by the procedure just described is said to be a sparsely-jumping sequence.

There are two very easy but important properties enjoyed by a sparsely-jumping sequence which we state as a lemma.

Lemma 7. Every sparsely-jumping sequence $\left(M_{n}\right)$ as above satisfies the following two properties:

(i) $\prod_{j=1}^{n} M_{j} \leq C n$ for some $C>0$;

(ii) $M_{k_{n}} \rightarrow \infty$ as $n \rightarrow \infty$.

Proof. Let $C=\prod_{j=1}^{\infty} u_{n}$. Since we have

$$
\prod_{j=1}^{n} M_{j}=\left(\prod_{j=1}^{n} u_{j}\right)\left(\prod_{j=1}^{m} \theta_{j}\right),
$$

where $m$ is largest with the property that $k_{m} \leq n$, it follows that

$$
\prod_{j=1}^{n} M_{j} \leq C r_{m} \leq C k_{m} \leq C n .
$$

At the same time, we have $M_{k_{n}}=\theta_{n} u_{k_{n}} \rightarrow \infty$ as $n \rightarrow \infty$.

Remark 3. Let $\mathscr{M}=\left(M_{n}\right)$ be a sparsely-jumping sequence of quasi-symmetric weights. Then property (i) in the above lemma implies that the cumulative distortion function of $\phi=\phi_{\mathscr{M}}$ satisfies the inequality $\kappa_{\phi}(n) \leq C n$ (this is simply because $\frac{1}{2}\left(1+M_{j}\right)<M_{j}$ for all $\left.j\right)$.

Theorem 3. Let $\mathscr{M}$ be a sparsely-jumping sequence of quasi-symmetric weights. Then the associated homeomorphism $\phi_{\mathscr{M}}:[0,1] \rightarrow[0,1]$ has modulus of continuity $\rho(t)=t \log (1 / t)$, and $\sigma_{\phi_{\mathscr{M}}}(x)=\infty$ for Lebesgue almost every $x$ in the unit interval.

Proof. Let us write $\phi=\phi_{\mathscr{M}}$ in this proof. The first assertion, concerning the modulus of continuity of $\phi$, follows from Lemma 5 . Hence we focus on the second assertion. We start by identifying a set of points $A_{\infty} \subseteq[0,1]$ where $\sigma_{\phi}$ blows up. 
Recall that any $x \in[0,1]$ can be written in binary as

$$
x=\sum_{j=1}^{\infty} \frac{x_{j}}{2^{j}},
$$

where $x_{j} \in\{0,1\}$ for each $j$, and the expansion is unique if we throw away a countable set of points. For each $n \geq 1$, let

$$
A_{n}=\left\{x \in(0,1): x_{k_{n}-1}=1 ; x_{k_{n}}=x_{k_{n}+1}=\cdots=x_{k_{n}+q_{n}}=0\right\} .
$$

Here, $\left(q_{n}\right)$ is the non-decreasing sequence of positive integers given by $q_{n}=1+$ $\left\lceil\log _{2} n\right\rceil$. Note that $q_{n} \rightarrow \infty$ but $\sum_{n=1}^{\infty} 2^{-q_{n}}=\infty$. Since we have $\mu\left(A_{n}\right)=2^{-q_{n}-2}$ for all $n$ (where $\mu$ denotes one-dimensional Lebesgue measure), it follows that

$$
\sum_{n=1}^{\infty} \mu\left(A_{n}\right)=\sum_{n=1}^{\infty} \frac{1}{2^{q_{n}+2}}=\infty .
$$

Moreover, the $A_{n}$ 's are independent events, as long as $q_{n}<k_{n+1}-1$ for all $n$, which is not a problem to assume since $k_{n+1} / k_{n} \rightarrow \infty$ (see the proof of Lemma 7 ). Therefore, by the Borel-Cantelli lemma, the set

$$
A_{\infty}=\limsup A_{n}=\bigcap_{k=1}^{\infty} \bigcup_{n \geq k} A_{n}
$$

has full Lebesgue measure. We will show that $\sigma_{\phi}(x)=\infty$ for all $x \in A_{\infty}$.

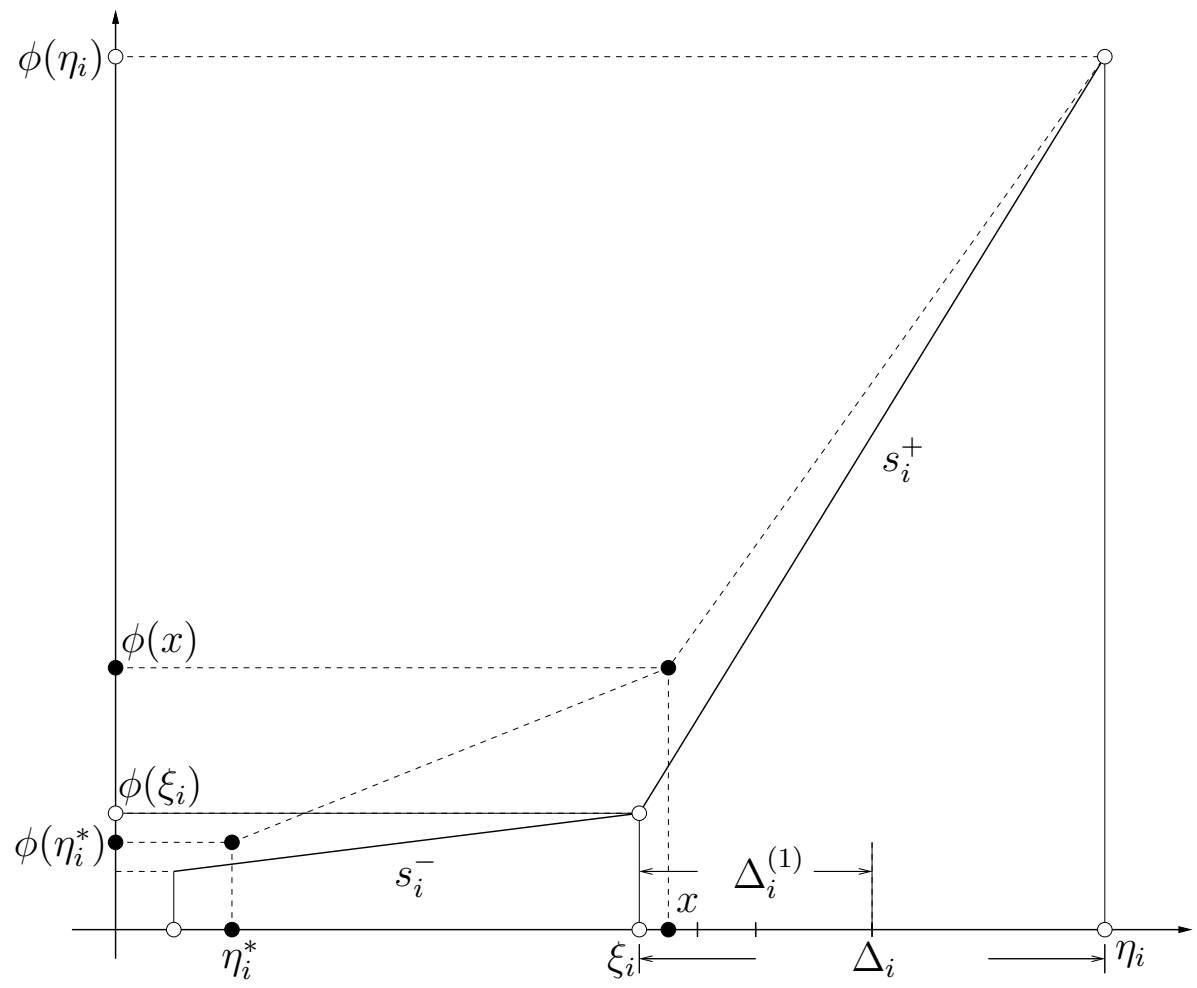

Figure 4. Forcing large quasi-symmetric distortion at $x \in A_{\infty}$. 
Given $x \in A_{\infty}$, let $n_{i} \rightarrow \infty$ be a sequence such that $x \in A_{n_{i}}$ for all $i$. Write

$$
\xi_{i}=\sum_{j=1}^{k_{n_{i}}-1} \frac{x_{j}}{2^{j}}
$$

so that

$$
\left|x-\xi_{i}\right|<\frac{1}{2^{k_{n_{i}}+q_{n_{i}}}} .
$$

Consider the atom of $\mathscr{P}_{k_{n_{i}-1}}$ containing $x$, namely $\Delta_{i}=\left[\xi_{i}, \eta_{i}\right]$, where $\eta_{i}=\xi_{i}+$ $2^{-k_{n_{i}}+1}$. For each $j \geq 0$, let $\Delta_{i}^{(j)}=\left[\xi_{i}, \xi_{i}+2^{-k_{n_{i}}-j+1}\right]$, so that

$$
\Delta_{i}=\Delta_{i}^{(0)} \supset \Delta_{i}^{(1)} \supset \Delta_{i}^{(2)} \supset \cdots \supset \Delta_{i}^{\left(q_{n_{i}}\right)} \supset\left[\xi_{i}, x\right] .
$$

We refer the reader to Figure 4. Our goal is to estimate

$$
\frac{\phi\left(\eta_{i}\right)-\phi(x)}{\phi(x)-\phi\left(\eta_{i}^{*}\right)}
$$

from below, where $\eta_{i}^{*}$ is the point symmetric to $\eta_{i}$ with respect to $x$, i.e., $\eta_{i}^{*}=2 x-\eta_{i}$. To do this, we first estimate $\phi(x)-\phi\left(\xi_{i}\right)$ from above. Since $\left[\xi_{i}, x\right] \subset \Delta_{i}^{\left(q_{n_{i}}\right)}$, we see using (12) that

$$
\left|\phi(x)-\phi\left(\xi_{i}\right)\right| \leq\left|\phi\left(\Delta_{i}^{\left(q_{n_{i}}\right)}\right)\right| \leq \prod_{j=k_{n_{i}}+1}^{k_{n_{i}}+q_{n_{i}}} \frac{2 M_{j}}{1+M_{j}}\left|\Delta_{i}^{\left(q_{n_{i}}\right)}\right| s_{i}^{+} .
$$

Here $s_{i}^{+}=D^{+} \phi_{k_{n_{i}}}\left(\xi_{i}\right)$. Since $\left|\Delta_{i}^{\left(q_{n_{i}}\right)}\right|=2^{-q_{n_{i}}}\left|\Delta_{i}\right|$, it follows that

$$
\left|\phi(x)-\phi\left(\xi_{i}\right)\right| \leq \prod_{j=k_{n_{i}}+1}^{k_{n_{i}}+q_{n_{i}}} \frac{M_{j}}{1+M_{j}}\left|\Delta_{i}\right| s_{i}^{+} .
$$

But by construction of $\mathscr{M}$, we have

$$
\prod_{j=k_{n_{i}}+1}^{k_{n_{i}}+q_{n_{i}}} \frac{M_{j}}{1+M_{j}}=\prod_{j=k_{n_{i}}+1}^{k_{n_{i}}+q_{n_{i}}} \frac{u_{j}}{1+u_{j}} \leq\left(\frac{2}{3}\right)^{q_{n_{i}}} .
$$

This yields the estimate

$$
\left|\phi(x)-\phi\left(\xi_{i}\right)\right| \leq \beta_{i}\left|\Delta_{i}\right| s_{i}^{+},
$$

where $\beta_{i}=\left(\frac{2}{3}\right)^{q_{n_{i}}}$.

Next, we estimate $\phi\left(\xi_{i}\right)-\phi\left(\eta_{i}^{*}\right)$, also from above. Let $\Delta_{i}^{*}$ the atom of $\mathscr{P}_{k_{n_{i}}-1}$ adjacent to $\Delta_{i}$ on the left. Then $\left[\eta_{i}^{*}, \xi_{i}\right] \subset \Delta_{i}^{*}$ and so

$$
\left|\phi\left(\xi_{i}\right)-\phi\left(\eta_{i}^{*}\right)\right| \leq\left|\phi\left(\Delta_{i}^{*}\right)\right|=\left|\Delta_{i}\right| s_{i}^{-},
$$

where $s_{i}^{-}=D^{-} \phi_{k_{n_{i}}}\left(\xi_{i}\right)$. Combining (15) with (16) we get

$$
\left|\phi(x)-\phi\left(\eta_{i}^{*}\right)\right| \leq\left(s_{i}^{-}+\beta_{i} s_{i}^{+}\right)\left|\Delta_{i}\right| .
$$

The next step is to estimate $\left|\phi\left(\eta_{i}\right)-\phi(x)\right|$ from below. But this is easy from (15), since

$$
\left|\phi\left(\eta_{i}\right)-\phi(x)\right|=\left|\phi\left(\Delta_{i}\right)\right|-\left|\phi(x)-\phi\left(\xi_{i}\right)\right| \geq\left(1-\beta_{i}\right)\left|\Delta_{i}\right| s_{i}^{+} .
$$


Here we have used that $\left|\phi\left(\Delta_{i}\right)\right|=s_{i}^{+}\left|\Delta_{i}\right|$. Putting (17) and (18) together, we deduce that

$$
\frac{\phi\left(\eta_{i}\right)-\phi(x)}{\phi(x)-\phi\left(\eta_{i}^{*}\right)} \geq \frac{\left(1-\beta_{i}\right) s_{i}^{+}}{s_{i}^{-}+\beta_{i} s_{i}^{+}}=\frac{\left(1-\beta_{i}\right)\left(s_{i}^{+} / s_{i}^{-}\right)}{1+\beta_{i}\left(s_{i}^{+} / s_{i}^{-}\right)} .
$$

But from (11) and the construction of $\left(M_{n}\right)$, we know that

$$
\frac{s_{i}^{+}}{s_{i}^{-}}=\frac{M_{k_{n_{i}}}}{M_{k_{n_{i}}-1}} \in\left[\frac{\theta_{n_{i}}}{2}, 2 \theta_{n_{i}}\right] \text {. }
$$

Hence we arrive at

$$
\frac{\phi\left(\eta_{i}\right)-\phi(x)}{\phi(x)-\phi\left(\eta_{i}^{*}\right)} \geq \frac{\frac{1}{2}\left(1-\beta_{i}\right) \theta_{n_{i}}}{1+2 \beta_{i} \theta_{n_{i}}} .
$$

Finally, we have by construction

$$
\theta_{n_{i}} \leq C_{1} \log n_{i} \leq C_{2} q_{n_{i}}
$$

and therefore

$$
\beta_{i} \theta_{n_{i}} \leq C_{2} q_{n_{i}}\left(\frac{2}{3}\right)^{q_{n_{i}}} \rightarrow 0 \text { as } i \rightarrow \infty
$$

This shows that the right hand side of (19) diverges to $\infty$. Hence $\sigma_{\phi}(x)=\infty$ for all $x \in A_{\infty}$, and the theorem is proved.

\section{Proof of Theorem 1}

The proof of theorem 1 is obtained by putting together the results of Theorems 2 and 3. Given any $\phi$ as in theorem 3, we know that its cumulative distortion function $\kappa_{\phi}$ satisfies the inequality $\kappa_{\phi}(n) \leq C n$ for some constant $C>0$. Hence, by Theorem 2, the Carleson boxes extension $f$ of $\phi$ to the unit square is a David homemorphism. Note that by construction $f$ agrees with the identity on the three sides of the unit square lying in the upper-half plane. We can extend $f$ to the infinite half-strip $[0,1] \times[0, \infty)$ by setting it to be the identity on $\{(x, y): 0 \leq x \leq 1, y \geq 1\}$. Now extend $\phi$ to the whole real line taking $\phi(x+n)=\phi(x)+n$ for all $x \in[0,1]$ and all $n \in \mathbf{Z}$. Likewise, extend $f$ to the whole half-plane $\mathbf{H}$ putting $f(z+n)=f(z)+n$ for all $z \in \mathbf{H}$ and all $n \in \mathbf{Z}$. Then the pointwise distortion of $\phi: \mathbf{R} \rightarrow \mathbf{R}$ is infinite almost everywhere, so that $\phi$ satisfies (a) of theorem 1. And it satisfies (b) of that theorem too, by lemma 5. Finally, its extension $f: \mathbf{H} \rightarrow \mathbf{H}$ is a David homeomorphism. This finishes the proof of Theorem 1.

Remark 4. In [Z], Zakeri defines the scalewise distortion of $\phi$ to be

$$
\rho_{\phi}(t)=\sup _{x \in \mathbf{R}} \max \left\{\frac{\phi(x+t)-\phi(x)}{\phi(x)-\phi(x-t)}, \frac{\phi(x)-\phi(x-t)}{\phi(x+t)-\phi(x)}\right\} .
$$

He proves that if

$$
\rho_{\phi}(t)=O\left(\log \frac{1}{t}\right)
$$

then $\phi$ extends to a David homeomorphism of the upper half-plane. Using this result, it is possible to prove Theorem 1 without having to invoke Theorem 2. All one needs to do is to first prove a lemma showing that the examples we constructed in $\S 4$ satisfy Zakeri's condition (20). Conversely, it is also possible to use Theorem 2 to produce an 
alternative proof of Zakeri's result (Zakeri's proof uses the Beurling-Ahlfors extension of $\phi)$.

Acknowledgements. The author wishes to thank Saeed Zakeri for posing the question, as well as for several stimulating mathematical discussions on these and related matters. He also thanks IMPA-RJ for its hospitality and generous support during the preparation of the manuscript.

\section{References}

[A] Ahlfors, L.: Lectures on quasiconformal mappings. - Van Nostrand, 1966.

[D] David, G.: Solutions de l'équation de Beltrami avec $\|\mu\|_{\infty}=1$. - Ann. Acad. Sci. Fenn. Math. $13,1988,25-70$.

[G] Gardiner, F.: Teichmüller theory and quadratic differentials. - John Wiley \& Sons, 1989.

[H] Haïssinsky, P.: Chirurgie parabolique. - C. R. Acad. Sci. Paris Sér. I Math. 327, 1998, 195-198.

[PZ] Petersen, C., and S. Zakeri: On the Julia set of a typical quadratic polynomial with a Siegel disk. - Ann. of Math. (2) 159, 2004, 1-52.

[Z] ZAKERI, S.: On boundary homeomorphisms of trans-quasiconformal maps of the disk. - Ann. Acad. Sci. Fenn. Math. 33, 2008, 241-260.

Received 25 March 2010 